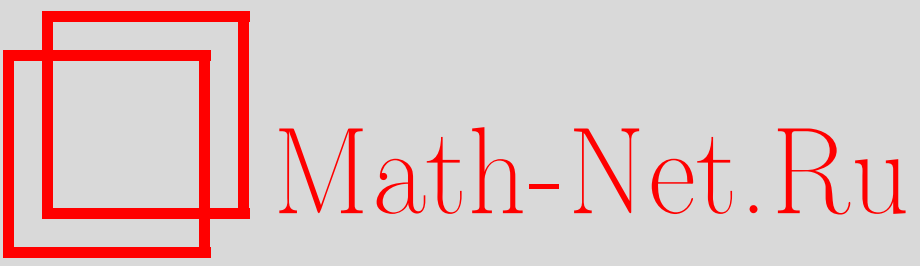

И. Н. Балаба, О слабо примитивных градуированных кольцах, УМН, 2001, том 56, выпуск 6, 139-140

DOI: https://doi.org/10.4213/rm456

Использование Общероссийского математического портала Math-Net.Ru подразумевает, что вы прочитали и согласны с пользовательским соглашением

http://www.mathnet.ru/rus/agreement

Параметры загрузки:

IP : 54.205 .225 .156

26 апреля 2023 г., 14:09:46 


\title{
О СЛАБО ПРИМИТИВНЫХ ГРАДУИРОВАННЫХ КОЛЬЦАХ
}

\author{
И. Н. БАЛАБА
}

Наиболее сильным обобщением классической теоремы плотности Джекобсона для первичных колец является теорема Зельмановича, опубликованная в [1], где было дано полное описание слабо примитивных колец. В работе дано описание градуированных слабо примитивных колец, основанное на градуированной версии теоремы Зельмановича [2].

Пусть $G$ - полугруппа, $H$ - группа и $A-(H, G)$-полигон. Следуя [3], назовем полигон $A_{G}$ строго точныц, если из того, что $a g_{1}=a g_{2}\left(a \in A, g_{1}, g_{2} \in G\right)$, следует, что $g_{1}=g_{2}$. Далее будем считать, что $A$ является строго точньм правым $G$-полигоном и левым $H$-полигоном, и полугруппа $G$ действует на нем инъективно, т.е. если $a_{1} g=a_{2} g$, то $a_{1}=a_{2}$.

Всюду далее $R-G$-градуированное кольцо, $M-A$-градуированный правьй $R$-модуль, $\operatorname{END}\left(M_{R}\right)-H$-градуированное кольцо эндоморфизмов, $h(R)$ - множество однородных элементов кольца $R$. Градуированным по группе $G$ кольцам посвящена монография [4], в [5] градуировка колец и модулей рассматривалась по полугруппе, а в [6] были рассмотрены модули, градуированные множеством, на котором действует группа $G$.

Напомним некоторые определения.

Модуль $M$ назьвается критически сжимаемым, если он вкладывается однородным гомоморфизмом в каждый свой ненулевой градуированный подмодуль и не вкладывается ни в какой свой собственный фактор-модуль. Градуированное кольцо называется слабо примитивным, если оно обладает точным критически сжимаемым градуированным модулем. Градуированное кольцо $R$ назьвается первичным, если для любых градуированных идеалов $I$ и $J$ кольца $R$ из $I J=0$ следует, что либо $I=0$, либо $J=0$.

ПрЕДЛОЖЕНИЕ 1. Градуированное слабо примитивное кольчо первично.

ДоКАЗАТЕЛЬСТВО. Пусть $M_{R}$-точный критически сжимаемьй градуированный $R$-модуль. Если $I \neq 0$ и $J \neq 0$ - градуированные идеалы кольца $R$, то $M I \neq 0$ и $M J \neq 0$. Так как $M_{R}$ сжимаем, то существует мономорфизм $f \in h\left(\operatorname{HOM}_{R}(M, M I)\right)$. Тогда $0 \neq f(M J)=f(M) J \subseteq$ $M I J$. Таким образом, $I J \neq 0$ и, следовательно, $R$ - первичное кольцо.

Пусть, далее, полигон ${ }_{H} A_{G}$ обладает следующим свойством: для любых $a_{1}, \ldots, a_{n} \in A$, $h \in H$ существуют такие элементы $g_{i j} \in G$, что $h a_{i}=a_{j} g_{i j}$.

Легко видеть, что в этом случае кольцо матриц $M_{n}(S)$ над $H$-градуированньм колшцом $S$ можно рассматривать как $G$-градуированное кольцо $M_{n}(S)\left(a_{1}, \ldots, a_{n}\right)$, где

$$
M_{n}(S)_{g}\left(a_{1}, \ldots, a_{n}\right)=\left(\begin{array}{cccc}
S_{a_{1} g a_{1}^{-1}} & S_{a_{1} g a_{2}^{-1}} & \ldots & S_{a_{1} g a_{n}^{-1}} \\
S_{a_{2} g a_{1}^{-1}} & S_{a_{2} g a_{2}^{-1}} & \ldots & S_{a_{2} g a_{n}^{-1}} \\
\vdots & \vdots & \ddots & \vdots \\
S_{a_{n} g a_{1}^{-1}} & S_{a_{n} g a_{2}^{-1}} & \ldots & S_{a_{n} g a_{n}^{-1}}
\end{array}\right)
$$

здесь через $a_{i} g a_{j}^{-1}$ обозначен такой элемент $h \in H$, для которого $h a_{j}=a_{i} g$, если же такого $h$ не существует, то положим $S_{a_{i} g a_{j}^{-1}}=0$.

ПредЛОЖенИЕ 2. Пусть $V$ - конечномерное $A$-градуированное векторное пространство над $H$-градуированным телом $\Delta$ с базисом $v_{1}, \ldots, v_{n}$, где $v_{i} \in V_{a_{i}}$. Tогда $\operatorname{End}(\Delta V)$ $=\operatorname{END}(\Delta V) \approx M_{n}(\Delta)\left(a_{1}, \ldots, a_{n}\right)$.

ДокаЗАтЕльСтво. Пусть $f \in \operatorname{End}(\Delta V)$, тогда $\left(v_{i}\right) f=\sum_{j=1}^{n} d_{i j} v_{j}$, где $d_{i j} \in \Delta$, и пусть $d_{i j}=\sum_{k=1}^{t i j} d_{i j}^{k}$, где $d_{i j}^{k} \in \Delta_{h_{i j}^{k}}$. В силу условий, наложенных на полигон, существуют такие $g_{i j}^{k} \in G$, что $h_{i j}^{k} a_{j}=a_{i} g_{i j}^{k}$. Легко проверить, что $\left(V_{a}\right) f \subseteq \sum_{g \in T} V_{a g}$, где $T=\left\{g_{i j}^{k}\right\}$, и, следовательно, $\operatorname{End}\left({ }_{\Delta} V\right)=\operatorname{END}(\Delta V)$. 
Если же $f \in \operatorname{END}\left({ }_{\Delta} V\right)_{g}$, то в представлении $\left(v_{i}\right) f=\sum_{i=1}^{n} d_{i j} v_{j}$ все элементы $d_{i j}$ можно считать однородными и $\operatorname{deg}\left(d_{i j}\right) a_{j}=a_{i} g$. Таким образом, отображение $\Phi: f \mapsto\left(d_{i j}\right)$ определяет изоморфизм градуированных колец $\operatorname{END}(\Delta V)$ и $M_{n}(\Delta)\left(a_{1}, \ldots, a_{n}\right)$.

Градуированное подкольцо $R$ колца $Q$ назовем градуированным правым порядком в $Q$, если $Q$ содержит единицу и для любого $q \in h(Q)$ найдутся такие $r, s \in h(R)$, причем $s$ - обратимый в $Q$, для которых $q=r s^{-1}$.

Теорема 3. Пусть $R$ - градуированное слабо примитивное кольцо. Тогда: либо (1) $R$ - правый порядок в матричном кольце $M_{n}(\Delta)$ для некоторого градуированного тела $\Delta$ (в этом случае $R$ содержит градуированное подкольцо, изоморфное $M_{n}(D)$ для некоторого градуированного правого порядка $D$ в $\Delta)$; либо (2) для каждого положительного челого числа п существуют градуированный правый порядок $D$ тела $\Delta u$ градуированное подкольчо кольца $R$, которое гомоморфно отображается на $M_{n}(D)$.

ДокАЗАТЕЛЬСтво. Пусть $M_{R}$-точный критически сжимаемый $A$-градуированный модул, тогда $\Delta=\operatorname{END}\left(\bar{M}_{R}\right)-H$-градуированное тело и $\bar{M}=\Delta M$ (здесь $\bar{M}$ - градуированная квазиинъективная оболочка модуля $M)$.

Предположим, что $\operatorname{dim}_{\Delta} \bar{M} \geqslant n$, тогда можно выбрать $\Delta$-линейно независимые элементы $m_{1}, \ldots, m_{n} \in h(M)\left(m_{i} \in M_{a_{i}}\right)$. Для каждого $i=1, \ldots, n$ положим $A_{i}=\bigcap_{j \neq i} m_{j}^{r}$. По теореме слабой плотности [7] $m_{i} A_{i} \neq 0$, тогда $N=\bigcap_{i=1}^{n} m_{i} A_{i} \neq 0$.

Положим $D_{h}=\left\{a \in \Delta_{h} \mid a M \subseteq N\right\}, D=\bigoplus_{h \in H} D_{h}$. Если $0 \neq \lambda \in h(\Delta)$, то $\lambda^{-1}(N) \cap$ $N \neq 0$ и существует мономорфизм $a \in h(D)$, для которого $a M \subseteq \lambda^{-1}(N) \cap N$. Следовательно, $0 \neq \lambda a \in D$ и $D$ - градуированный правый порядок в $\Delta$.

Пусть далее $W_{n}=\sum_{i=1}^{n} D m_{i}, W_{n}^{\prime}=\sum_{i=1}^{n} D^{1} m_{i}\left(D^{1}\right.$ - теоретико-множественное объединение $D$ и множества рациональных чисел) и $\varphi \in \operatorname{HOM}_{D}\left(W^{\prime}, W\right)_{g}$. Тогда $\left(m_{i}\right) \varphi=\sum_{j=1}^{n} d_{i j} m_{j}$ для подходящих $d_{i j} \in h(D)$. Посколшку $d_{i j} m_{j} \in h(N)$, то $d_{i j} m_{j}=m_{i} r_{i j}$ для некоторых $r_{i j} \in\left(A_{i}\right)_{g}$. Таким образом, $\left(m_{i}\right) \varphi=\sum_{j=1}^{n} m_{i} r_{i j}=m_{i} r_{i}$, где $r_{i}=\sum_{j=1}^{n} r_{i j} \in\left(A_{i}\right)_{g}$. Положив $r=\sum_{i=1}^{n} r_{i}$, получим, что $\left(m_{i}\right) \varphi=m_{i} r$ для всех $i=1, \ldots, n$.

Легко видеть, что $S=\left\{s \in R \mid W^{\prime} s \subseteq W\right\}$ является градуированным подкольцом кольца $R$. Отображение $\left.s \mapsto r_{s}\right|_{W^{\prime}}$, где $r_{s}$ - правое умножение на элемент $s$, определяет гомоморфизм кольца $S$ на $\operatorname{HOM}_{D}\left(W^{\prime}, W\right) \approx M_{n}(D)\left(a_{1}, \ldots, a_{n}\right)$ с ядром $K=\left\{r \in R \mid W^{\prime} r=0\right\}$.

Если же $\operatorname{dim}_{\Delta} \bar{M}=n$, то элементы $m_{1}, \ldots, m_{n} \in h(M)$ образуют базис $\Delta \bar{M}$ и $K=0$. Таким образом, $R$ содержит градуированное подкольо, изоморфное $M_{n}(D)\left(a_{1}, \ldots, a_{n}\right)$ для некоторого градуированного правого порядка $D$ в $\Delta$.

Полагая $U=U^{\prime}=\bar{M}$ в условии (3) теоремы из [2], получим, что для любого $f \in \operatorname{END}\left({ }_{\Delta} \bar{M}\right)$ существуют такие $r, s \in R$, что $\left.(f r-s)\right|_{U}=0$ и $\left.r\right|_{U^{\prime}}$ является градуированным автоморфизмом. Таким образом, $R$ является правым порядком в колшце $\operatorname{END}\left({ }_{\Delta} \bar{M}\right)=\operatorname{End}\left({ }_{\Delta} \bar{M}\right) \approx M_{n}(\Delta)$.

В заключение приношу искреннюю благодарность А. В. Михалёву за полезные обсуждения.

\section{СПИСОК ЛИТЕРАТУРЫ}

[1] J. Zelmanowitz // Comm. Algebra. 1981. V. 9. №1. Р. 23-45. [2] С. В. Зеленов // УМH. 2001. T. 56. № 3. C. 167-168. [3] M. Kilp, U. Krauner, A. V. Mikhalev. Monoids, Acts and Categories. Berlin: de Gruyter, 2000. [4] C. Năstăsescu, F. van Oystaeyen. Graded Ring Theory. Amsterdam: North-Holland, 1982. [5] G. Abrams, C. Menini, A. del Rio // Comm. Algebra. 1994. V. 22 № 13. P. 5343-5388. [6] C. Năstăsescu, S. Raianu, F. van Oystaeyen // Math. Z. 1990. V. 203. № 4. P. 605-627. [7] С. В. Зеленов // Труды 6-х математических чтений МГСУ. М.: МГСУ, 1999. С. 107-110. 